\title{
Robust Event-based Stabilization of Periodic Orbits for Hybrid Systems: Application to an Underactuated 3D Bipedal Robot
}

\author{
Kaveh Akbari Hamed ${ }^{1}$ and J. W. Grizzle ${ }^{2}$
}

\begin{abstract}
The first return map or Poincaré map can be viewed as a discrete-time dynamical system evolving on a hyper surface that is transversal to a periodic orbit; the hyper surface is called a Poincaré section. The Poincaré map is a standard tool for assessing the stability of periodic orbits in non-hybrid as well as hybrid systems. In addition, it can be used for stabilization of periodic orbits if the underlying dynamics of the system depends on a set of parameters that can be updated by a feedback law when trajectories cross the Poincaré section. This paper addresses an important practical obstacle that arises when designing feedback laws on the basis of the Jacobian linearization of the Poincaré map. In almost all practical cases, the Jacobians must be estimated numerically, and when the underlying dynamics presents a wide range of time scales, the numerical approximations of the first partial derivatives are sufficiently inaccurate that controller tuning is very difficult. Here, a robust control formalism is proposed whereby a convex set of approximations to the Jacobian linearization is systematically generated and a stabilizing controller is designed through two appropriate sets of linear matrix inequalities (LMIs). The result is illustrated on a walking gait of a 3D underactuated bipedal robot.
\end{abstract}

\section{INTRODUCTION}

Robotic bipedal locomotion is being profitably studied with hybrid models [1]-[4]. Steady-state walking and running gaits correspond to periodic orbits in the hybrid model. The most fundamental tool for analyzing the existence and stability of periodic orbits for hybrid systems is the method of Poincaré's sections [5]-[7], [1, Chap. 4], [8]. This method presents an equivalence between stability properties of transversal periodic orbits for the hybrid system and those of the corresponding equilibrium points of the discrete-time system defined by the Poincaré return map on a transversal hyperplane, referred to as the Poincaré section.

The approach of Poincaré's sections can also be employed to stabilize periodic orbits for hybrid systems [9]-[11]. In this case, the underlying dynamics of the system depends on a set of adjustable parameters that can be updated by an eventbased law when the trajectories cross the Poincaré section. In [12]-[15], linear event-based controllers have been successfully designed on the basis of the Jacobian linearization of the Poincaré map. In these cases, the dynamic models had up to 18 states and the Jacobians were approximated numerically. We have been pursuing similar event-based control designs for ATRIAS, a new 3D bipedal robot with series-compliant actuators [16], [17] and have encountered

\footnotetext{
*This work was supported by DARPA Contract W91CRB-11-1-0002.

${ }^{1},{ }^{2}$ K. Akbari Hamed and J. W. Grizzle are with the Electrical Engineering and Computer Science Department of the University Michigan, Ann Arbor, MI, USA, \{kavehah, grizzle\}@umich.edu
}

problems obtaining adequately robust control designs. The source of the difficulty seems to be that the large springs used for energy storage in the series-compliant actuators render the robot's dynamics numerically stiff, which in turn leads to inaccurate estimates of the Jacobians when using central differences.

In this paper, we regard the numerically-determined Jacobians as uncertain estimates of the true Jacobians and apply methods from robust linear control to design the event-based controller. In particular, the main contribution of the paper is to present two LMI-based stabilization approaches for a class of hybrid systems, composed of $N \geq 1$ phases, to reduce the sensitivity of the closed-loop system to numerical errors and also to parametric uncertainties in the $N$-step Poincaré map. Parameterized and continuously differentiable continuousphase feedback laws are employed at the first level of the control scheme. At the second level, the parameters of the continuous-phase feedback laws are updated in an event-based manner to stabilize the periodic orbit for the closed-loop hybrid system. The results of the paper are then illustrated on ATRIAS which has 13 degrees of freedom (DOFs) and 7 degrees of underactuation (DOUs) during the single support phase of walking.

\section{Robust Orbital Stabilization of Periodic ORBITS FOR HYBRID SYSTEMS}

This section addresses robust stabilization of periodic orbits for a class of hybrid systems, arising from systems with impulse effects [18], [19] and composed of $N \geq 1$ continuous phases, against numerical errors and parametric uncertainties in the $N$-step Poincare map. The results of this section will be used in Sections III and IV for ATRIAS 2.1. To define a hybrid system with $N$ continuous phases, let us consider the ordinary differential equations $\dot{x}_{i}=$ $f_{i}\left(x_{i}\right)+g_{i}\left(x_{i}\right) u_{i}, i=1, \cdots, N$ defined on the state spaces $\mathcal{X}_{i}$, where $\mathcal{X}_{i}$ are embedded submanifolds of $\mathbb{R}^{n_{i}}$ for some $n_{i}>0$. The control inputs $u_{i}, i=1, \cdots, N$ take values in $\mathcal{U}_{i}$, in which $\mathcal{U}_{i} \subset \mathbb{R}^{m_{i}}$ represents the set of admissible control inputs during phase $i$ and $0<m_{i}<n_{i}$. It is supposed that the following hypothesis is satisfied for the continuous phases.

H1) The vector fields $f_{i}$ and columns of $g_{i}, i=1, \cdots, N$ are continuously differentiable (i.e., $\mathcal{C}^{1}$ ).

We assume that the phases are executed in the fixed order $1 \rightarrow 2 \rightarrow \cdots \rightarrow N \rightarrow 1$. In particular, we define the index of next phase function as $\mu:\{1, \cdots, N\} \rightarrow\{1, \cdots, N\}$ by

$$
\mu(i):= \begin{cases}i+1, & i=1, \cdots, N-1 \\ 1, & i=N .\end{cases}
$$


Next, let $\mathcal{S}_{i \rightarrow \mu(i)}$ be the switching hypersurface in $\mathcal{X}_{i}$ on which the transition from phase $i$ to phase $\mu(i)$ (i.e., from $\mathcal{X}_{i}$ to $\mathcal{X}_{\mu(i)}$ ) occurs, according to the transition map $\Delta_{i \rightarrow \mu(i)}$ : $\mathcal{S}_{i \rightarrow \mu(i)} \rightarrow \mathcal{X}_{\mu(i)}$ as $x_{\mu(i)}^{+}=\Delta_{i \rightarrow \mu(i)}\left(x_{i}^{-}\right)$. Here, the superscripts "-" and "+" denote the state of the hybrid system just before and after the corresponding discrete transition. It is assumed that $\mathcal{S}_{i \rightarrow \mu(i)}$ can be expressed as the zero level set of the $\mathcal{C}^{1}$ function $H_{i \rightarrow \mu(i)}: \mathcal{X}_{i} \rightarrow \mathbb{R}$, i.e.,

$$
\mathcal{S}_{i \rightarrow \mu(i)}:=\left\{x_{i} \in \mathcal{X}_{i} \mid H_{i \rightarrow \mu(i)}\left(x_{i}\right)=0\right\}
$$

for which the following hypotheses are satisfied.

H2) For every $x_{i} \in \mathcal{S}_{i \rightarrow \mu(i)}, \partial H_{i \rightarrow \mu(i)} / \partial x_{i}\left(x_{i}\right) \neq 0$ so that $\mathcal{S}_{i \rightarrow \mu(i)}$ is a $n_{i}-1$ dimensional embedded submanifold of $\mathcal{X}_{i}$.

H3) The transition maps $\Delta_{i \rightarrow \mu(i)}, i=1, \cdots, N$ are $\mathcal{C}^{1}$. Furthermore, $\Delta_{i \rightarrow \mu(i)}\left(\mathcal{S}_{i \rightarrow \mu(i)}\right) \cap \mathcal{S}_{\mu(i) \rightarrow \mu \circ \mu(i)}=\emptyset$ to prevent the chattering of solutions, where $\mu \circ \mu(i):=$ $\mu(\mu(i))$ and " $\circ$ " represents the function composition.

The hybrid system with $N$ continuous phases denoted by $\mathcal{H}\left(\Sigma_{1}, \cdots, \Sigma_{N}\right)$ is then defined as

$$
\begin{aligned}
& \Sigma_{1}: \begin{cases}\dot{x}_{1}=f_{1}\left(x_{1}\right)+g_{1}\left(x_{1}\right) u_{1}, & x_{1}^{-} \notin \mathcal{S}_{1 \rightarrow 2} \\
x_{2}^{+}=\Delta_{1 \rightarrow 2}\left(x_{1}^{-}\right), & x_{1}^{-} \in \mathcal{S}_{1 \rightarrow 2}\end{cases} \\
& \vdots \\
& \Sigma_{N}: \begin{cases}\dot{x}_{N}=f_{N}\left(x_{N}\right)+g_{N}\left(x_{N}\right) u_{N}, & x_{N}^{-} \notin \mathcal{S}_{N \rightarrow 1} \\
x_{1}^{+}=\Delta_{N \rightarrow 1}\left(x_{N}^{-}\right), & x_{N}^{-} \in \mathcal{S}_{N \rightarrow 1} .\end{cases}
\end{aligned}
$$

A solution of the hybrid system (1) is constructed by piecing together the trajectories of the flows $\dot{x}_{i}=f_{i}\left(x_{i}\right)+$ $g_{i}\left(x_{i}\right) u_{i}, i=1, \cdots, N$ such that the transitions take place when these flows intersect the switching hypersurfaces $\mathcal{S}_{i \rightarrow \mu(i)}$. Next, let $\mathcal{A}_{i} \subset \mathbb{R}^{p_{i}}, i=1, \cdots, N$ be finitedimensional parameter spaces, referred to as stabilizing parameter spaces, for some $p_{i}>0$. Define a family of parameterized feedback laws $u_{i}: \mathcal{X}_{i} \times \mathcal{A}_{i} \rightarrow \mathcal{U}_{i}$ by the following piecewise policy

$$
u_{i}\left(x_{i}, \alpha_{i}\right):= \begin{cases}\operatorname{fcn}_{i}\left(x_{i}, \alpha_{i}^{*}\right), & s_{i}\left(x_{i}\right)<s_{\mathrm{th}, i} \\ \operatorname{fcn}_{i}\left(x_{i}, \alpha_{i}\right), & s_{i}\left(x_{i}\right) \geq s_{\mathrm{th}, i} .\end{cases}
$$

In (2), fcn f $_{i}: \mathcal{X}_{i} \times \mathcal{A}_{i} \rightarrow \mathcal{U}_{i}$ for $i=1, \cdots, N$ are $\mathcal{C}^{1}$ functions with respect to $\left(x_{i}, \alpha_{i}\right)$ on $\mathcal{X}_{i} \times \mathcal{A}_{i}$. Moreover, $\alpha_{i}^{*} \in \mathcal{A}_{i}, i=$ $1, \cdots, N$ denote a set of nominal parameters. $s_{i}: \mathcal{X}_{i} \rightarrow \mathbb{R}$ by $s_{i}\left(x_{i}\right)$ is a $\mathcal{C}^{1}$ real-valued function and $s_{\text {th }, i} \in \mathbb{R}$ represents a threshold value of $s_{i}$ to be determined. Associated with $s_{i}$ and $s_{\text {th, } i}$, we can define the event-based control surface during phase $i$ as follows

$$
\mathcal{S}_{\mathrm{th}, i}:=\left\{x_{i} \in \mathcal{X}_{i} \mid s_{i}\left(x_{i}\right)=s_{\mathrm{th}, i}\right\} .
$$

It is assumed that the following hypothesis is satisfied.

H4) On the surface $\mathcal{S}_{\mathrm{th}, i}, i=1, \cdots, N$,

(i) $\operatorname{fcn}_{i}\left(x_{i}, \alpha_{i}^{*}\right)=\mathrm{fcn}_{i}\left(x_{i}, \alpha_{i}\right)$

(ii) $\frac{\partial \mathrm{fcn}_{i}}{\partial x_{i}}\left(x_{i}, \alpha_{i}^{*}\right)=\frac{\partial \mathrm{fcn}_{i}}{\partial x_{i}}\left(x_{i}, \alpha_{i}\right)$

(iii) $\frac{\partial \mathrm{fcn}_{i}}{\partial \alpha_{i}}\left(x_{i}, \alpha_{i}^{*}\right)=0_{m_{i} \times p_{i}}$ which in turn implies that $u_{i}$ is $\mathcal{C}^{1}$ with respect to $\left(x_{i}, \alpha_{i}\right)$ on $\mathcal{X}_{i} \times \mathcal{A}_{i}$.

Remark 1: According to the piecewise policy in (2), the stabilizing parameter $\alpha_{i}$ is employed after the threshold value $s_{\text {th }, i}$ (see hypothesis H6). The physical intuition behind this is that human push recovery is mainly done by changing the step length at the end of the current step.

Next, for every $\alpha_{1} \in \mathcal{A}_{1}, \cdots, \alpha_{N} \in \mathcal{A}_{N}$, the parameterized hybrid system $\mathcal{H}\left(\Sigma_{\mathrm{cl}, 1, \alpha_{1}}, \cdots, \Sigma_{\mathrm{cl}, N, \alpha_{N}}\right)$ can be expressed as

$$
\begin{aligned}
& \Sigma_{\mathrm{cl}, 1, \alpha_{1}}: \begin{cases}\dot{x}_{1}=f_{\mathrm{cl}, 1}\left(x_{1}, \alpha_{1}\right), & x_{1}^{-} \notin \mathcal{S}_{1 \rightarrow 2} \\
x_{2}^{+}=\Delta_{1 \rightarrow 2}\left(x_{1}^{-}\right), & x_{1}^{-} \in \mathcal{S}_{1 \rightarrow 2}\end{cases} \\
& \vdots \\
& \Sigma_{\mathrm{cl}, N, \alpha_{N}}: \begin{cases}\dot{x}_{N}=f_{\mathrm{cl}, N}\left(x_{N}, \alpha_{N}\right), & x_{N}^{-} \notin \mathcal{S}_{N \rightarrow 1} \\
x_{1}^{+}=\Delta_{N \rightarrow 1}\left(x_{N}^{-}\right), & x_{N}^{-} \in \mathcal{S}_{N \rightarrow 1},\end{cases}
\end{aligned}
$$

in which $f_{\mathrm{cl}, i}\left(x_{i}, \alpha_{i}\right):=f_{i}\left(x_{i}\right)+g_{i}\left(x_{i}\right) u_{i}\left(x_{i}, \alpha_{i}\right)$. Throughout this paper, we shall assume that the following hypotheses are met.

H5) Associated with the nominal stabilizing parameters $\alpha_{i}^{*} \in \mathcal{A}_{i}, i=1, \cdots, N$, the parameterized hybrid system $\mathcal{H}\left(\Sigma_{\mathrm{cl}, 1, \alpha_{1}^{*}}, \cdots, \Sigma_{\mathrm{cl}, N, \alpha_{N}^{*}}\right)$ has a nontrivial periodic orbit $\mathcal{O}=\cup_{i=1}^{N} \mathcal{O}_{i}$ transversal to the switching manifolds $\mathcal{S}_{i \rightarrow \mu(i)}$ and also to the event-based control surfaces $\mathcal{S}_{\text {th }, i}, i=1, \cdots, N$, where $\mathcal{O}_{i}:=\mathcal{O} \cap \mathcal{X}_{i}$. In particular, $\left\{x_{i}^{*}\right\}:=\overline{\mathcal{O}}_{i} \cap \mathcal{S}_{i \rightarrow \mu(i)}$ and $\left\{x_{\mathrm{th}, i}^{*}\right\}:=$ $\overline{\mathcal{O}}_{i} \cap \mathcal{S}_{\text {th }, i}$ for $i=1, \cdots, N$ are singletons and

$$
\begin{aligned}
& \partial H_{i \rightarrow \mu(i)} / \partial x_{i}\left(x_{i}^{*}\right) f_{\mathrm{cl}, i}\left(x_{i}^{*}, \alpha_{i}^{*}\right) \neq 0 \\
& \partial s_{i} / \partial x_{i}\left(x_{\mathrm{th}, i}^{*}\right) f_{\mathrm{cl}, i}\left(x_{\mathrm{th}, i}^{*}, \alpha_{i}^{*}\right) \neq 0,
\end{aligned}
$$

where $\overline{\mathcal{O}}_{i}$ is the set closure of $\mathcal{O}_{i}$.

H6) On the periodic orbit $\mathcal{O}, s_{i}\left(x_{i}\right)$ is a strictly increasing function of time. Moreover in (2), $s_{\mathrm{th}, i}$ can be chosen in the interval $\left(s_{i}^{*+}, s_{i}^{*-}\right)$, where $s_{i}^{*+}$ and $s_{i}^{*-}$ are the initial and final values of $s_{i}$ during phase $i$ of $\mathcal{O}$.

Next, for a given initial condition $x_{i}(0) \in \mathcal{X}_{i}$ and a given stabilizing parameter $\alpha_{i} \in \mathcal{A}_{i}$, let $\varphi_{i}\left(t ; x_{i}(0), \alpha_{i}\right)$ represent the solution of the parameterized closed-loop differential equation $\dot{x}_{i}=f_{\mathrm{cl}, i}\left(x_{i}, \alpha_{i}\right)$ with the initial condition $x_{i}(0)$ over the maximal interval of existence. Suppose that $\Gamma_{i}$ is a hypersurface in $\mathcal{X}_{i}$. Associated with the pair $\left(x_{i}(0), \alpha_{i}\right) \in$ $\mathcal{X}_{i} \times \mathcal{A}_{i}$, the flow of $\dot{x}_{i}=f_{\mathrm{cl}, i}\left(x_{i}, \alpha_{i}\right)$, evaluated on $\Gamma_{i}$, can be expressed as $\mathcal{F}_{i}^{\Gamma_{i}}: \mathcal{X}_{i} \times \mathcal{A}_{i} \rightarrow \Gamma_{i}$ by

$$
\mathcal{F}_{i}^{\Gamma_{i}}\left(x_{i}(0), \alpha_{i}\right):=\varphi_{i}\left(T_{i}^{\Gamma_{i}}\left(x_{i}(0), \alpha_{i}\right) ; x_{i}(0), \alpha_{i}\right),
$$

in which $T_{i}^{\Gamma_{i}}: \mathcal{X}_{i} \times \mathcal{A}_{i} \rightarrow \mathbb{R}_{\geq 0}$ represents the time of the first impact of $\varphi_{i}$ with the hypersurface $\Gamma_{i}$, i.e.,

$$
T_{i}^{\Gamma_{i}}\left(x_{i}(0), \alpha_{i}\right):=\inf \left\{t \geq 0 \mid \varphi_{i}\left(t ; x_{i}(0), \alpha_{i}\right) \in \Gamma_{i}\right\} .
$$

Now we are in a position to present the $N$-step Poincaré map. For this goal, without loss of generality, let $\mathcal{S}_{\text {th, } 1}$ be the Poincaré section during phase 1 . Then, the $N$-step Poincaré map $\mathcal{P}: \mathcal{S}_{\text {th }, 1} \times \mathcal{A}_{1} \times \cdots \mathcal{A}_{N} \rightarrow \mathcal{S}_{\text {th }, 1}$ can be defined as

$$
\begin{aligned}
& \mathcal{P}\left(x_{1}(0), \alpha_{1}, \cdots, \alpha_{N}\right):=
\end{aligned}
$$

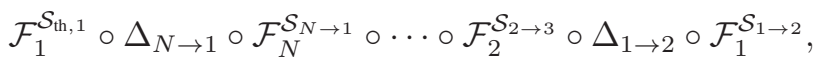


where $x_{1}(0) \in \mathcal{S}_{\text {th,1 }}$ and $\alpha_{i}, i=1, \cdots, N$ is employed for $s_{i}\left(x_{i}\right) \geq s_{\mathrm{th}, i}$ during phase $i$. According to the construction procedure, $x_{\mathrm{th}, 1}^{*}$ (see hypothesis H5) is the fixed point of $\mathcal{P}$, i.e., $\mathcal{P}\left(x_{\mathrm{th}, 1}^{*}, \alpha_{1}^{*}, \cdots, \alpha_{N}^{*}\right)=x_{\mathrm{th}, 1}^{*}$. Linearization of the $N$-step Poincaré map around $\left(x_{\mathrm{th}, 1}^{*}, \alpha_{1}^{*}, \cdots, \alpha_{N}^{*}\right)$ yields the following discrete-time system with the state space $\mathcal{S}_{\text {th }, 1}$ which is updated in $N$-step fashion

$$
\delta x_{1}[k+1]=A \delta x_{1}[k]+B\left[\begin{array}{c}
\delta \alpha_{1}[k] \\
\vdots \\
\delta \alpha_{N}[k]
\end{array}\right], \quad k=0,1, \cdots,
$$

where $\delta x_{1}[k]:=x_{1}[k]-x_{\mathrm{th}, 1}^{*}, \delta \alpha_{i}[k]:=\alpha_{i}[k]-\alpha_{i}^{*}$ and

$$
\begin{aligned}
A & :=\frac{\partial \mathcal{P}}{\partial x_{1}(0)}\left(x_{\mathrm{th}, 1}^{*}, \alpha_{1}^{*}, \cdots, \alpha_{N}^{*}\right) \\
B & :=\frac{\partial \mathcal{P}}{\partial\left(\alpha_{1}, \cdots, \alpha_{N}\right)}\left(x_{\mathrm{th}, 1}^{*}, \alpha_{1}^{*}, \cdots, \alpha_{N}^{*}\right) .
\end{aligned}
$$

Due to the high dimension and range of time scales in models describing walking of $3 \mathrm{D}$ robots like ATRIAS, there may be significant errors during numerical differentiating of the $N$ step Poincare map to obtain the Jacobian matrices $A$ and $B$. This complicates the design of the gain matrix $K$ to stabilize $(A, B)$. To overcome this problem, we present two LMIbased gain design approaches. In particular, assume that $A(\varepsilon)$ and $B(\varepsilon)$ represent numerical approximations of the Jacobian matrices $A$ and $B$ which are obtained based on the two-point difference approach with the perturbation value $\varepsilon$. Next, we present the following Theorem.

Theorem 1 (Robust Stabilization of $\mathcal{O}$ ): Assume that hypotheses H1-H6 are satisfied for the open-loop hybrid model (1). Let $\mathcal{F P}^{A}:=\left\{\varepsilon_{p}^{A}\right\}_{p=1}^{M_{A}}$ and $\mathcal{F} \mathcal{P}^{B}:=\left\{\varepsilon_{q}^{B}\right\}_{q=1}^{M_{B}}$ be two feasible sets of perturbations used for numerical differentiating of the $N$-step Poincaré map to obtain $A$ and $B$ matrices, respectively. Define $A_{p}:=A\left(\varepsilon_{p}^{A}\right)$ for $p=1, \cdots, M_{A}$ and $B_{q}:=B\left(\varepsilon_{q}^{B}\right)$ for $q=1, \cdots, M_{B}$. Moreover, assume that due to numerical problems or parametric uncertainty, the Jacobian matrices $A$ and $B$ are unknown and belong to some convex polytope $\mathbf{A}$ and $\mathbf{B}$. In particular, $A \in \mathbf{A}:=$ $\operatorname{conv}\left\{A_{1}, \cdots, A_{M_{A}}\right\}$ and $B \in \mathbf{B}:=\operatorname{conv}\left\{B_{1}, \cdots, B_{M_{B}}\right\}$. Set up the following two optimization problems,

$$
\begin{aligned}
\eta_{\min }:= & \min _{\eta, S, W} \eta \\
\text { s.t. } & F_{p, q}:=\left[\begin{array}{cc}
-S & A_{p} S+B_{q} W \\
\star & -S
\end{array}\right] \leq \eta I \\
& p=1, \cdots, M_{A}, q=1, \cdots, M_{B}
\end{aligned}
$$

and

$$
\begin{aligned}
& \xi_{\min }:=\min _{\xi, P_{p, q}, G, L} \xi \\
& \text { s.t. } \quad J_{p, q}:=-\left[\begin{array}{cc}
P_{p, q} & A_{p} G+B_{q} L \\
\star & G+G^{\prime}-L
\end{array}\right] \leq \xi I \\
& \quad p=1, \cdots, M_{A}, q=1, \cdots, M_{B} .
\end{aligned}
$$

Then, the following statements are true.

1) (LMI stability with known Lyapunov matrix): If $\eta_{\min }<$ 0 in the optimization problem (9), the $N$-step update policy

$$
\left[\begin{array}{c}
\alpha_{1}[k] \\
\vdots \\
\alpha_{N}[k]
\end{array}\right]=\left[\begin{array}{c}
\alpha_{1}^{*} \\
\vdots \\
\alpha_{N}^{*}
\end{array}\right]-K \delta x_{1}[k]
$$

employed on the surface $\mathcal{S}_{\text {th,1 }}$ during phase 1, exponentially and robustly stabilizes the periodic orbit $\mathcal{O}$ for the closed-loop hybrid model of walking, where $K:=-W S^{-1}$. Moreover, $V\left(\delta x_{1}\right):=\delta x_{1}^{\prime} S^{-1} \delta x_{1}$ is the corresponding Lyapunov function for (7).

2) (LMI stability with unknown Lyapunov matrix): If $\xi_{\min }<0$ in problem (10), the update policy (11) exponentially and robustly stabilizes the periodic orbit $\mathcal{O}$ for the closed-loop hybrid model of walking, where $K:=-L G^{-1}$. Moreover, there exists $P \in$ $\operatorname{conv}\left\{P_{p, q} \mid p=1, \cdots, M_{A}, q=1, \cdots, M_{B}\right\}$ such that $V\left(\delta x_{1}\right):=\delta x_{1}^{\prime} P \delta x_{1}$ is the corresponding Lyapunov function for (7).

Proof: Hypotheses H1, H3, H4 and H5 imply that the $N$-step Poincaré map is $\mathcal{C}^{1}$ with respect to $\left(x_{\mathrm{th}, 1}, \alpha_{1}, \cdots, \alpha_{N}\right)$ in an open neighborhood of $\left(x_{\mathrm{th}, 1}^{*}, \alpha_{1}^{*}, \cdots, \alpha_{N}^{*}\right)$. Consequently, the Jacobian matrices $A$ and $B$ in (8) are well-defined. Moreover from H6, there is an open neighborhood $\mathcal{N}$ of $\mathcal{O}$, such that in $\mathcal{N} \backslash \mathcal{O}$, the feedback laws $u_{i}, i=1, \cdots, N$ are piecewise in the sense that the solutions intersect the threshold manifold $\mathcal{S}_{\text {th }, i}$. Thus, the stabilizing parameter $\alpha_{i}$ can be employed during phase $i$, which is a necessary condition for $\frac{\partial \mathcal{P}}{\partial \alpha_{i}}() \neq$.0 .

Part (1): $\eta_{\min }<0$ implies that $F_{p, q}<0$ for $p=$ $1, \cdots, M_{A}$ and $q=1, \cdots, M_{B}$. Next for a given $A \in$ $\operatorname{conv}\left\{A_{1}, \cdots, A_{M_{A}}\right\}$ and $B \in \operatorname{conv}\left\{B_{1}, \cdots, B_{M_{B}}\right\}$, there are $a_{p} \geq 0, p=1, \cdots, M_{A}$ and $B_{q} \geq 0, q=1, \cdots, M_{B}$ such that $A=\sum_{p=1}^{M_{A}} a_{p} A_{p}$ and $B=\sum_{q=1}^{M_{B}} b_{q} B_{q}$. Moreover, $\sum_{p=1}^{M_{A}} a_{p}=1$ and $\sum_{q=1}^{M_{B}} b_{q}=1$. Thus,

$$
\sum_{p=1}^{M_{A}} \sum_{q=1}^{M_{B}} a_{p} b_{q} F_{p, q}=\left[\begin{array}{cc}
-S & A S+B W \\
\star & -S
\end{array}\right]<0 .
$$

Using Schur's Lemma, the LMI problem (12) is equivalent to (i) $-S<0$ and (ii)

$$
-S-\left(S A^{\prime}+W^{\prime} B^{\prime}\right)(-S)^{-1}(A S+B W)<0 .
$$

Defining $P:=S^{-1}$ and $K=-W S^{-1}$ translates this into $P>0$ and $(A-B K)^{\prime} P(A-B K)-P<0$. Thus, $\delta x_{1}=$ $0_{n_{1}-1}$ is exponentially and robustly stable for (7). Finally, Theorem 4.7 of [1] completes the proof of the exponential stability of $\mathcal{O}$ for the closed-loop hybrid model.

Part (2): $\xi_{\text {min }}<0$ yields $J_{p, q}<0$ for $p=1, \cdots, M_{A}$ and $q=1, \cdots, M_{B}$. The exponential stability of $\delta x_{1}=$ $0_{n_{1}-1}$ for (7) then follows from [20]. Furthermore, $P=$ $\sum_{p=1}^{M_{A}} \sum_{q=1}^{M_{B}} a_{p} b_{q} P_{p, q}$ is the corresponding Lyapunov matrix. Similar to part (1), applying Theorem 4.7 of [1] completes the proof.

\section{BACKGROUND: HYBRID MODEL FOR 3D WALKING OF ATRIAS}

This section extends the results of Section II to a 26-dimensional hybrid model describing 3D walking by 
ATRIAS 2.1. During the single support phase (one point foot on the ground), ATRIAS is an underactuated mechanical system with 13 DOFs and 7 DOUs. The double support phase (both point feet on the ground) of walking is assumed to be instantaneous. ATRIAS exploits four mechanical springs to drive the legs in the sagittal plane for energy efficiency. In particular, the springs absorb the energy during the impact and release it within the step. To develop the hybrid model of walking by ATRIAS, a convenient set of configuration variables is presented. To achieve this goal, let us attach the torso frame rigidly to the torso link with the origin on its center of mass (COM). Next, the rotation of the torso frame with respect to the world frame can be expressed as $R_{\mathrm{T}}^{0}:=R_{z}\left(q_{z \mathrm{~T}}\right) R_{y}\left(q_{y \mathrm{~T}}\right) R_{x}\left(q_{x \mathrm{~T}}\right)$, where $R_{x}, R_{y}$ and $R_{z}$ are the basis rotations about the $x, y$ and $z$ axes, respectively. Moreover, $q_{z \mathrm{~T}}, q_{y \mathrm{~T}}$ and $q_{x \mathrm{~T}}$ are the yaw, roll and pitch angles of the robot (see Fig. 1). In the frontal plane of the robot, the angles of the right and left hips relative to the torso link are expressed by $q_{3 \mathrm{R}}$ and $q_{3 \mathrm{~L}}$, respectively, where the subscripts "R" and "L" denote the right and left. Furthermore, in the sagittal plane of the robot, the angles of the shin and thigh links relative to the torso are given by $q_{1 \mathrm{R}}$ and $q_{2 \mathrm{R}}$ for the right leg and $q_{1 \mathrm{~L}}$ and $q_{2 \mathrm{~L}}$ for the left leg (see Fig. 1). The motors in the sagittal plane are series-elastic actuators and connected through springs. Consequently, we denote the corresponding angle of the output shafts (i.e., position of springs) by $q_{g r 1 \mathrm{R}}, q_{g r 2 \mathrm{R}}, q_{g r 1 \mathrm{~L}}$ and $q_{g r 2 \mathrm{~L}}$. This completes the configuration variables for the single support phase of ATRIAS as $q:=\left(q_{u}^{\prime}, q_{a}^{\prime}\right) \in \mathcal{Q}$, in which $q_{u}$ and $q_{a}$ are unactuated and actuated components of $q$, i.e., $q_{u}:=\left(q_{z \mathrm{~T}}, q_{y \mathrm{~T}}, q_{x \mathrm{~T}}, q_{1 \mathrm{R}}, q_{2 \mathrm{R}}, q_{1 \mathrm{~L}}, q_{2 \mathrm{~L}}\right)^{\prime}$ and $q_{a}:=$ $\left(q_{g r 1 \mathrm{R}}, q_{g r 2 \mathrm{R}}, q_{3 \mathrm{R}}, q_{g r 1 \mathrm{~L}}, q_{g r 2 \mathrm{~L}}, q_{3 \mathrm{~L}}\right)^{\prime}$ and $\mathcal{Q}$ is the configuration space. The torque input of the mechanical system can also be expressed as $u:=\left(u_{1 \mathrm{R}}, u_{2 \mathrm{R}}, u_{3 \mathrm{R}}, u_{1 \mathrm{~L}}, u_{2 \mathrm{~L}}, u_{3 \mathrm{~L}}\right)^{\prime} \in$ $\mathbb{R}^{6}$, where $u_{1 \mathrm{R}}, u_{2 \mathrm{R}}, u_{3 \mathrm{R}}, u_{1 \mathrm{~L}}, u_{2 \mathrm{~L}}$ and $u_{3 \mathrm{~L}}$ are motor torques to drive $q_{g r 1 \mathrm{R}}, q_{g r 2 \mathrm{R}}, q_{3 \mathrm{R}}, q_{g r 1 \mathrm{~L}}, q_{g r 2 \mathrm{~L}}$ and $q_{g r 3 \mathrm{~L}}$ through fixed gear ratio systems, respectively.

\section{A. Continuous phases of walking}

To simplify the analysis and to present the main idea, we will omit the stance phase index $i \in\{\mathrm{R}, \mathrm{L}\}$. Next, the evolution of the mechanical system during continuous phases of walking can be expressed as

$$
\begin{aligned}
D(q) \ddot{q} & +C(q, \dot{q}) \dot{q}+G(q)+\Gamma_{\text {spring }}(q)+\Gamma_{\text {damper }}(q, \dot{q}) \\
& +\Gamma_{\text {friction }}(q, \dot{q})=B_{\text {input }} u
\end{aligned}
$$

where $D(q) \in \mathbb{R}^{13 \times 13}$ is the positive-definite mass-inertia matrix and $C(q, \dot{q}) \dot{q} \in \mathbb{R}^{13}$ represents the Coriolis and centrifugal terms. Furthermore, $G(q) \in \mathbb{R}^{13}, \Gamma_{\text {spring }}(q) \in$ $\mathbb{R}^{13}$ and $\Gamma_{\text {damper }}(q, \dot{q}) \in \mathbb{R}^{13}$ are the terms associated with the gravity, spring and damping forces of the compliant elements, respectively. $\Gamma_{\text {friction }}(q, \dot{q}) \in \mathbb{R}^{13}$ denotes the rotational friction with respect to the yaw motion about the stance leg end. Finally, $B_{\text {input }} \in \mathbb{R}^{13 \times 6}$ is the input matrix with the property rank $B_{\text {input }}=6$.

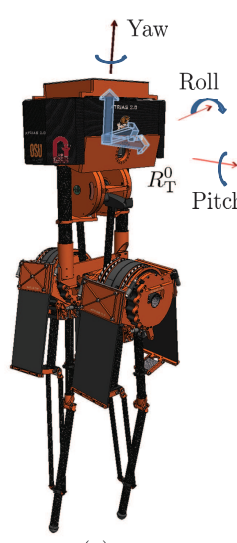

(a)

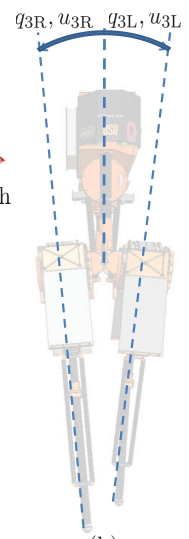

(b)

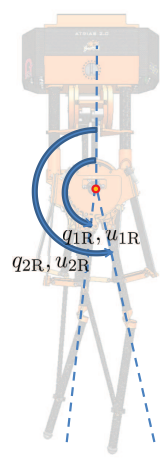

(c)
Fig. 1: ATRIAS 2.1, a bipedal prototype. (a) Rotation matrix $R_{T}^{0}$ together with the yaw, roll and pitch angles. (b) Hip angles and motor torques in the frontal plane. (c) Shin and thigh angles with respect to the torso and corresponding motor torques in the sagittal plane.

\section{B. Discrete transitions of walking}

Following the developments of reference [14], to present the discrete transition models, conservation of momentum together with stationary position for the swing leg end while preserving the yaw rotation about the swing leg end during impact yields instantaneous impact maps $\left(q^{+}, \dot{q}^{+}\right)=$ $\Delta_{\mathrm{R} \rightarrow \mathrm{L}}\left(q^{-}, \dot{q}^{-}\right)$and $\left(q^{+}, \dot{q}^{+}\right)=\Delta_{\mathrm{L} \rightarrow \mathrm{R}}\left(q^{-}, \dot{q}^{-}\right)$.

\section{Hybrid model of walking}

Let us define the state vector of the mechanical system as $x:=\left(q^{\prime}, \dot{q}^{\prime}\right)^{\prime} \in \mathcal{X}$, in which $\mathcal{X}:=T \mathcal{Q}$ is the 26-dimensional tangent bundle of the configuration space $\mathcal{Q}$. Next, the evolution of the mechanical system during walking by ATRIAS can be expressed by a hybrid system composed of two continuous phases to represent the right and left stance phases and two discrete transitions between the continuous phases to denote the right to left and left to right instantaneous impact maps. In particular,

$$
\begin{aligned}
& \Sigma_{\mathrm{R}}: \begin{cases}\dot{x}=f_{\mathrm{R}}(x)+g_{\mathrm{R}}(x) u, & x^{-} \notin \mathcal{S}_{\mathrm{R} \rightarrow \mathrm{L}} \\
x^{+}=\Delta_{\mathrm{R} \rightarrow \mathrm{L}}\left(x^{-}\right), & x^{-} \in \mathcal{S}_{\mathrm{R} \rightarrow \mathrm{L}}\end{cases} \\
& \Sigma_{\mathrm{L}}: \begin{cases}\dot{x}=f_{\mathrm{L}}(x)+g_{\mathrm{L}}(x) u, & x^{-} \notin \mathcal{S}_{\mathrm{L} \rightarrow \mathrm{R}} \\
x^{+}=\Delta_{\mathrm{L} \rightarrow \mathrm{R}}\left(x^{-}\right), & x^{-} \in \mathcal{S}_{\mathrm{L} \rightarrow \mathrm{R}} .\end{cases}
\end{aligned}
$$

In (14), the input-affine system $\dot{x}=f_{i}(x)+g_{i}(x) u$ represents the evolution of the mechanical system during phase $i \in\{\mathrm{R}, \mathrm{L}\}$. Moreover, $\mathcal{S}_{i \rightarrow j}$ denotes the 25-dimensional switching manifold during the transition from phase $i$ to phase $j$, where $i \neq j \in\{\mathrm{R}, \mathrm{L}\}$. We note that $\mathcal{S}_{i \rightarrow j}:=$ $\left\{x \in \mathcal{X} \mid p_{\mathrm{sw}}^{z}(q)=0\right\}$, in which $p_{\mathrm{sw}}^{z}: \mathcal{Q} \rightarrow \mathbb{R}$ is the vertical component (i.e., $z$ component) of the swing leg end Cartesian coordinates. The impact maps are then expressed by $\Delta_{i \rightarrow j}: \mathcal{S}_{i \rightarrow j} \rightarrow \mathcal{X}, i \neq j \in\{\mathrm{R}, \mathrm{L}\}$. For later purposes, 
we assume that $\mathcal{O}:=\mathcal{O}_{\mathrm{R}} \cup \mathcal{O}_{\mathrm{L}}$ is a periodic orbit of the hybrid model (14) to be stabilized.

\section{HZD AND LMI-BASED CONTROL STRATEGY FOR EXPONENTIAL AND ROBUST STABILIZATION}

This section presents a dual-level and time-invariant control strategy based on virtual constraints [7], hybrid zero dynamics (HZD) [1, Chap. 6] and Theorem 1 to exponentially and robustly stabilize periodic orbits for 3D walking by ATRIAS. At the first level of the control strategy, 6 virtual constraints are defined as parameterized holonomic output functions in both the sagittal and frontal planes of the mechanical system and imposed to be zero by using the within-stride controller. The within-stride controller is a parameterized time-invariant and continuously differentiable feedback law which creates a family of parameterized attractive and forward invariant zero dynamics manifolds. On these manifolds, the mechanical system has 7 DOFs and its evolution can be expressed by the unactuated components. Moreover, the evolution of the controlled variables can be expressed in terms of a strictly increasing holonomic quantity referred to as the phasing term, defined in the sagittal plane. Virtual constraints coordinate the links of the robot during $3 \mathrm{D}$ walking. In particular, four components of the virtual constraints are defined in the sagittal plane of the robot and they are capable to stabilize periodic orbits for the planar model of walking by ATRIAS. The two remaining components are defined in the frontal plane to synchronize the stance hip angle as well as the distance between the horizontal components of the swing leg end and robot's COM with the phasing term in the sagittal plane.

At the second level of the control strategy, the parameters of the virtual constraints are updated by two discrete-time loops. The first loop updates the parameters of the output function to render the family of the zero dynamics manifolds hybrid invariant under the closed-loop system. This restricts the state of the hybrid model to a 14-dimensional reducedorder hybrid system referred to as the HZD. According to Theorem 1, the second loop exponentially stabilizes the corresponding fixed point of the two-step Poincaré return map on the basis of a two-step event-based correction.

\section{A. Within-stride controller}

During continuous phases of walking, let us define the following parameterized holonomic output function

$$
\begin{aligned}
y\left(x ; \alpha_{\mathrm{hi}}, \alpha_{\mathrm{st}}\right) & :=h\left(q ; \alpha_{\mathrm{hi}}, \alpha_{\mathrm{st}}\right) \\
& :=h_{\mathrm{nom}}(q)-h_{\mathrm{hi}}\left(\theta ; \alpha_{\mathrm{hi}}\right)-h_{\mathrm{st}}\left(\theta ; \alpha_{\mathrm{st}}\right) \\
& :=\underbrace{h_{c}(q)-h_{d}(\theta)}_{h_{\mathrm{nom}}(q)}-h_{\mathrm{hi}}\left(\theta ; \alpha_{\mathrm{hi}}\right)-h_{\mathrm{st}}\left(\theta ; \alpha_{\mathrm{st}}\right) .
\end{aligned}
$$

In $(15), y\left(x ; \alpha_{\mathrm{hi}}, \alpha_{\mathrm{st}}\right):=h\left(q ; \alpha_{\mathrm{hi}}, \alpha_{\mathrm{st}}\right) \in \mathbb{R}^{6}$ represents the holonomic output function to be regulated. Furthermore, $\alpha_{\mathrm{hi}}$ and $\alpha_{\mathrm{st}}$ are the parameters of the output function corresponding to the hybrid invariance and stabilization issues. $h_{\text {nom }}(q) \in \mathbb{R}^{6}$ is a nominal output function vanishing on the desired periodic orbit $\mathcal{O}$. In particular, $h_{\text {nom }}(q):=h_{c}(q)-$ $h_{d}(\theta)$, where $h_{c}(q) \in \mathbb{R}^{6}$ denotes the controlled variables consisting of a set of holonomic quantities to be controlled. The desired evolution of the controlled variables on the periodic orbit $\mathcal{O}$ can be given by the vector $h_{d}(\theta) \in \mathbb{R}^{6}$ in terms of the phasing term $\theta(q)$. For the ATRIAS structure, $\theta$ is chosen as the angle of the virtual leg with respect to the ground, where the virtual leg is defined as a virtual line in the sagittal plane of the robot which connects the stance leg end to the stance hip joint. We note that on the periodic walking motion $\mathcal{O}, \theta$ is a strictly increasing function of time (hypothesis H6). $h_{d}(\theta)$ can be obtained by regressing the controlled variables $h_{c}(q)$ versus $\theta$ on $\mathcal{O}$. Furthermore, $h_{\mathrm{hi}}\left(\theta ; \alpha_{\mathrm{hi}}\right)$ and $h_{\mathrm{st}}\left(\theta ; \alpha_{\mathrm{st}}\right)$ are additive terms to create hybrid invariant zero dynamics manifolds and to stabilize $\mathcal{O}$, respectively. These terms are zero on the periodic orbit. The parameters of the hybrid invariance and stabilizing terms, i.e., $\alpha_{\mathrm{hi}} \in \mathcal{A}_{\mathrm{hi}}$ and $\alpha_{\mathrm{st}} \in \mathcal{A}_{\mathrm{st}}$, are updated in a step-by-step fashion, in which $\mathcal{A}_{\mathrm{hi}}$ and $\mathcal{A}_{\text {st }}$ represent the corresponding finite-dimensional parameter spaces.

The within-stride controller is obtained based on inputoutput linearization. To this end, differentiating the output function $y$ with respect to time and along the trajectories of the continuous phases results in

$$
\ddot{y}=L_{g} L_{f} h\left(q ; \alpha_{\mathrm{hi}}, \alpha_{\mathrm{st}}\right) u+L_{f}^{2} h\left(q, \dot{q} ; \alpha_{\mathrm{hi}}, \alpha_{\mathrm{st}}\right) .
$$

Consequently, the parameterized feedback law

$$
\begin{aligned}
u\left(x ; \alpha_{\mathrm{hi}}, \alpha_{\mathrm{st}}\right) & =-\left(L_{g} L_{f} h\left(q ; \alpha_{\mathrm{hi}}, \alpha_{\mathrm{st}}\right)\right)^{-1}\left(L_{f}^{2} h\left(q, \dot{q} ; \alpha_{\mathrm{hi}}, \alpha_{\mathrm{st}}\right)\right. \\
& \left.+\frac{K_{D}}{\epsilon} L_{f} h\left(q, \dot{q} ; \alpha_{\mathrm{hi}}, \alpha_{\mathrm{st}}\right)+\frac{K_{P}}{\epsilon^{2}} h\left(q, \dot{q} ; \alpha_{\mathrm{hi}}, \alpha_{\mathrm{st}}\right)\right)
\end{aligned}
$$

yields the following output dynamics

$$
\ddot{y}+\frac{K_{D}}{\epsilon} \dot{y}+\frac{K_{P}}{\epsilon^{2}} y=0,
$$

where $K_{P}=k_{p} I_{6 \times 6}>0, K_{D}=k_{d} I_{6 \times 6}>0$ and $\epsilon>0$. The zero dynamics manifold associated with the output function (15) can be expressed as the following 14dimensional attractive and forward invariant manifold

$$
\mathcal{Z}_{\alpha_{\mathrm{hi}}, \alpha_{\mathrm{st}}}:=\left\{x \in \mathcal{X} \mid y\left(x ; \alpha_{\mathrm{hi}}, \alpha_{\mathrm{st}}\right)=L_{f} y\left(x: \alpha_{\mathrm{hi}}, \alpha_{\mathrm{st}}\right)=0_{6}\right\} .
$$

\section{B. Hybrid invariance}

In order to render the zero dynamics manifolds hybrid invariant, the parameters of the hybrid invariance term $h_{\text {hi }}\left(\theta ; \alpha_{\text {hi }}\right)$ are updated at the beginning of each step to satisfy

$$
\begin{aligned}
h_{\mathrm{hi}}\left(\theta^{+} ; \alpha_{\mathrm{hi}}\right) & =h_{\text {nom }}\left(q^{+}\right) \\
\frac{\partial h_{\mathrm{hi}}}{\partial \theta}\left(\theta^{+} ; \alpha_{\mathrm{hi}}\right) \dot{\theta}^{+} & =\frac{\partial h_{\mathrm{nom}}}{\partial q}\left(q^{+}\right) \dot{q}^{+},
\end{aligned}
$$

which in turn implies that ${ }^{1}$

$$
y\left(x^{+} ; \alpha_{\mathrm{hi}}, \alpha_{\mathrm{st}}\right)=L_{f} y\left(x^{+} ; \alpha_{\mathrm{hi}}, \alpha_{\mathrm{st}}\right)=0_{6} .
$$

\footnotetext{
${ }^{1}$ According to (2) and hypothesis $\mathrm{H} 4$, the stabilizing term $h_{\mathrm{st}}\left(\theta ; \alpha_{\mathrm{st}}\right)$ is designed to be zero at the beginning of the step.
} 
In this paper, it is assumed that $h_{\mathrm{hi}}\left(\theta ; \alpha_{\mathrm{hi}}\right)$ is a fifth-order polynomial on the interval $\left[\theta^{+}, \theta_{\mathrm{hi}}\right]$, where $\theta_{\mathrm{hi}}:=\frac{1}{2}\left(\theta^{+}+\theta_{f}\right)$ is the hybrid invariance threshold and $\theta_{f}$ is the final value of $\theta$ on $\mathcal{O}$. Moreover, $h_{\mathrm{hi}}\left(\theta ; \alpha_{\mathrm{hi}}\right)$ is identically zero for $\theta>$ $\theta_{\text {hi }}$ while continuity of position, velocity and acceleration is met at $\theta=\theta_{\mathrm{hi}}$. Consequently, the family of zero dynamics manifolds $\mathbf{Z}:=\left\{\mathcal{Z}_{\alpha_{\mathrm{hi}}, \alpha_{\mathrm{st}}} \mid \alpha_{\mathrm{hi}} \in \mathcal{A}_{\mathrm{hi}}, \alpha_{\mathrm{st}} \in \mathcal{A}_{\mathrm{st}}\right\}$ is hybrid invariant under the flow of the closed-loop hybrid system.

\section{Stabilization}

To exponentially stabilize the desired periodic orbit $\mathcal{O}$ based on Theorem 1, the parameters of the stabilizing term $h_{\mathrm{st}}\left(\theta ; \alpha_{\mathrm{st}}\right)$ are updated in a two step manner. In particular, we assume that $h_{\mathrm{st}}\left(\theta^{+} ; \alpha_{\mathrm{st}}\right)=0_{6}$ and $\frac{\partial}{\partial \theta} h_{\mathrm{st}}\left(\theta^{+} ; \alpha_{\mathrm{st}}\right) \dot{\theta}^{+}=0_{6}$ to preserve the hybrid invariance condition of Section IVB. Moreover, to define a Poincaré section for stabilization issue, we consider a stabilization threshold value $\theta_{\mathrm{st}}$ with the property $\theta_{\text {hi }}<\theta_{\text {st }}<\theta_{f}$ and suppose that $h_{\text {st }}\left(\theta ; \alpha_{\text {st }}\right) \equiv 0_{6}$ for $\theta<\theta_{\mathrm{st}}$. Furthermore, $h_{\mathrm{st}}\left(\theta ; \alpha_{\mathrm{st}}\right)$ is assumed to be a third order polynomial with parameters $\alpha_{\text {st }}$ for $\theta>\theta_{\text {st }}$ while twice differentiability is satisfied at $\theta_{\mathrm{st}}$. Next, define the eventbased control surface $\mathcal{S}_{\text {th }}$ as the level set $\theta(q)=\theta_{\text {st }}$, i.e., $\mathcal{S}_{\text {th }}:=\left\{x \in \mathcal{X} \mid \theta(q)=\theta_{\text {st }}\right\}$ (see (3)). According to the construction procedure of the hybrid invariance and stabilizing terms, the stabilization term is active for $\theta(q) \geq \theta_{\text {st }}$ while $h$ in (15) is twice continuously differentiable (i.e., $\mathcal{C}^{2}$ ) with respect to its arguments. This together with the withinstride controller (16) implies hypothesis H4. Furthermore, since $h_{d}(\theta)$ is obtained by regressing the controlled variables on $\mathcal{O}$ and the additive terms are zero on $\mathcal{O}$, H5 is met. By defining $s_{i}=\theta$, it can be concluded that $\mathrm{H} 6$ is satisfied and consequently, Theorem 1 can be employed.

\section{Closed-loop simulation results}

In order to confirm the analytical results obtained in the paper, this section presents simulation of the closed-loop hybrid system. For this goal, the sagittal components of the controlled variables during the right stance phase are defined as $h_{c, 1}(q)=\frac{1}{2}\left(q_{g r 1 \mathrm{R}}+q_{g r 2 \mathrm{R}}\right), h_{c, 2}(q)=\frac{1}{2}\left(q_{g r 1 \mathrm{~L}}+q_{g r 2 \mathrm{~L}}\right)$, $h_{c, 3}(q)=q_{g r 2 \mathrm{R}}-q_{g r 1 \mathrm{R}}$ and $h_{c, 4}(q)=q_{g r 2 \mathrm{~L}}-q_{g r 1 \mathrm{~L}}$. We have observed that these components can stabilize periodic orbits for the 2D model of ATRIAS ${ }^{2}$. Here, we stabilize a periodic orbit $\mathcal{O}$ on which the robot has the average speed $1(\mathrm{~m} / \mathrm{s})$. The event-based surface $\mathcal{S}_{\text {th }}$ is also defined in $\frac{2}{3}$ of the nominal gait. Let $x_{\mathrm{sw}}(q)$ and $x_{\mathrm{cm}}(q)$ represent the horizontal Cartesian coordinates of the swing leg end and robot's COM in the frontal plane with respect to the stance leg end, respectively. If the frontal components of $h_{c}$ are defined as $h_{c, 5}(q)=q_{3 \mathrm{R}}$ and $h_{c, 6}(q)=x_{\mathrm{sw}}(q)-x_{\mathrm{cm}}(q)$, the three biggest eigenvalues of the averaged Jacobian $A_{\text {ave }}:=$ $\frac{1}{M_{A}} \sum_{p=1}^{M_{A}} A_{p}$ are $\lambda_{1}=1.1745, \lambda_{2}=1.0000$ and $\lambda_{3}=$ 0.8987 . Here the feasible set of perturbations are $\mathcal{F} \mathcal{P}^{A}=$ $\left\{10^{-5} \times\{1,5\}, 10^{-4} \times\{1,5\}, 10^{-3} \times\{1: 1: 15\}\right\}$ and $\mathcal{F} \mathcal{P}^{B}=\left\{10^{-5} \times\{1,5\}, 10^{-4} \times\{1: 1: 10\}, 5 \times 10^{-3}, 10^{-2} \times\right.$ $\{1,5\}\}$. Figure 2 illustrates the variation of $\left\|A_{p}\right\|_{\infty}$ and

\footnotetext{
${ }^{2}$ During the left stance phase, a similar definition can be presented by swapping the role of the right and left legs.
}
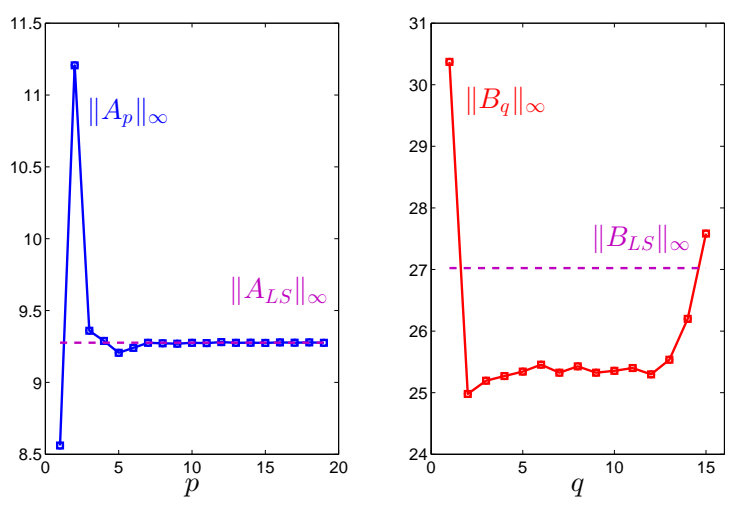

Fig. 2: Plot of $\left\|A_{p}\right\|_{\infty}$ and $\left\|B_{q}\right\|_{\infty}$ (solid curves) versus $p$ and $q$ together with $\left\|A_{L S}\right\|_{\infty}$ and $\left\|B_{L S}\right\|_{\infty}$ (dashed curves).

$\left\|B_{q}\right\|_{\infty}$, obtained based on the two point difference approach, versus $p=1, \cdots, M_{A}$ and $q=1, \cdots, M_{B}$, respectively. It also presents $\left\|A_{L S}\right\|_{\infty}$ and $\left\|B_{L S}\right\|_{\infty}$, obtained based on the least square approach over the sets $\mathcal{F} \mathcal{P}^{A}$ and $\mathcal{F} \mathcal{P}^{B}$.

1) Inadequacy of Discrete $L Q R$ approach: Figure 2 illustrates that there is extensive variability in the numerical estimates of the Jacobian linearization of the Poincare map. We tried designing DLQR controllers for several pairs $\left(A_{p}, B_{q}\right)$ in the "flat region" of the figure, and all of them failed. We then emabarked on a tedious iterative process, exhaustively searching over the collection of pairs $\left(A_{p}, B_{q}\right)$ for a range of weights $\left\{Q_{r}\right\}_{r=1}^{M_{Q}}$ and $\left\{R_{l}\right\}_{l=1}^{M_{R}}$, solving for the DLQR gain. A stabilizing gait was found using this approach, but it had a small basin of attraction. In particular, this stabilizing gain could only ensure stability for an external horizontal disturbance in the frontal plane with the magnitude of 10 (N) acts on the robot's COM over $50 \%$ of the gait.

2) LMI approach: Each of the LMI approaches in Theorem 1 resulted in stabilizing solutions. To show the robustness of the resulting closed-loop system against external disturbances, the simulation of the closed-loop system is started at the end of the left stance phase of $\mathcal{O}$. Next during the 21st step, a horizontal force in the frontal plane with the magnitude $60(\mathrm{~N})$ is applied to the robot's COM over $50 \%$ of the step. Figure 3 depicts the phase portraits of the yaw, roll and pitch angles and also the COM and foot steps in the $x y$ plane during 60 consecutive steps of walking. Convergence to the periodic orbit and yaw (heading) stability is clear.

\section{CONCLuSion}

During stabilization of 3D dynamic walking and running locomotion for legged robots with high degrees of freedom and underactuation, there are important practical problems arising from numerical approximation of the Jacobian matrices for the corresponding Poincaré return map. Due to the existence of a wide range of time scales in the underlying nonlinear dynamics, the Jacobian matrices, which are mainly calculated using numerical differentiation algorithms, such as the two point difference or the least square approaches, are sensitive to the perturbation values used during calculation. 


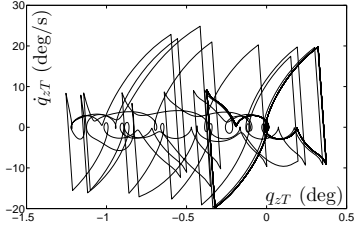

(a)

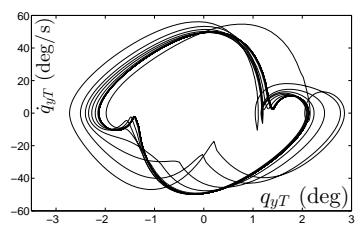

(b)

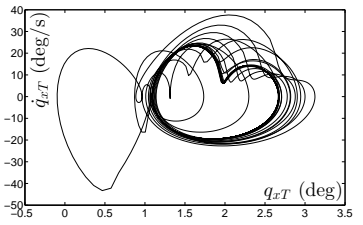

(c)

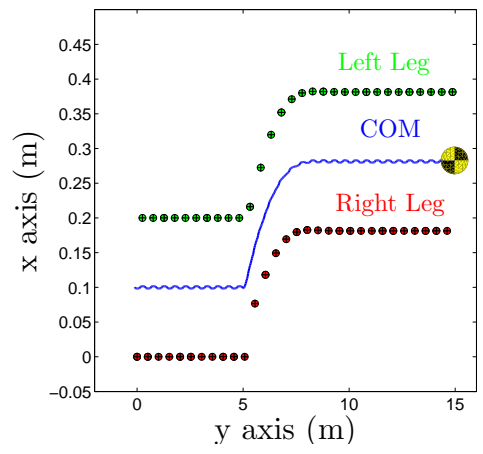

(d)

Fig. 3: Phase portraits for (a) the yaw, (b) roll, and (c) pitch angles together with (d) the COM and foot steps during 60 consecutive steps of walking. At the 21 st step, a horizontal force in the frontal plane with the magnitude $60(\mathrm{~N})$ is applied to the robot's COM over $50 \%$ of the step.

This numerical sensitivity, which can be formulated as uncertainty in the Jacobian matrices or the Poincaré return map, complicates the design of stabilizing controllers for periodic orbits. To overcome this problem, the paper has presented an LMI-based nonlinear control scheme to exponentially and robustly stabilize periodic orbits for a class of hybrid systems, namely systems with impulse effects. In particular, a robust control formalism has been presented whereby a convex set of approximations of the Jacobian linearization is generated and the feedback law is designed through two sets of LMIs. The results of the paper were illustrated on a 3D dynamic walking motion of a novel bipedal robot, ATRIAS 2.1. The resulting controller had much greater disturbance rejection capability than a corresponding DLQR controller.

The results of this paper were developed on the basis of the $N$-step Poincaré return map which in turn yields a $N$ step update law for stabilizing parameters. It would be very interesting to develop one-step robust and stabilizing update laws. It is conjectured that this will result in larger domains of attraction for the periodic orbit.

\section{REFERENCES}

[1] E. R. Westervelt, J. W. Grizzle, C. Chevallereau, J. H. Choi, and B. Morris, Feedback Control of Dynamic Bipedal Robot Locomotion, Boca Raton: CRC Press, June 2007.

[2] J. W. Grizzle, C. Chevallereau, A. D. Ames, and R. W. Sinnet, "3D bipedal robotic walking: Models, feedback control, and open problems," Proceedings of the IFAC Symposium on Nonlinear Control Systems, Bologna, September 2010.

[3] A. D. Ames, R. Sinnet and E. Wendel, "Three-dimensional kneed bipedal walking: A hybrid geometric approach," Proceedings of the 12th International Conference on Hybrid Systems: Computation and Control, Lecture Notes in Computer Science (HSCC 2009), vol. 5469, pages 16-30, April 2009.

[4] K. Akbari Hamed, N. Sadati, W. A. Gruver, and G. A. Dumont, "Stabilization of periodic orbits for planar walking with non-instantaneous double support phase," IEEE Transactions on Systems, Man, and Cybernetics, Part A, vol. 42, issue 3, pp. 685-706, May 2012.

[5] V. I. Arnold, Geometrical Methods in the Theory of Ordinary Differential Equations, Springer-Verlag, 1988.

[6] T. S. Parker and L. O. Chua, Practical Numerical Algorithms for Chaotic Systems, Springer-Verlag, New York, 1989.

[7] J. W. Grizzle, G. Abba, and F. Plestan, "Asymptotically stable walking for biped robots: Analysis via systems with impulse effects," IEEE Transactions on Automatic Control, vol. 46, issue 1, pp. 51-64, January 2001.

[8] W. M. Haddad and V. Chellaboina, Nonlinear Dynamical Systems and Control: A Lyapunov-Based Approach, Princeton, NJ, Princeton University Press, 2008.

[9] J. W. Grizzle, E. R. Westervelt, and C. Canudas, "Event-based PI control of an underactuated biped walker," Proceedings of the 2003 IEEE International Conference on Decision and Control, Maui, HI, pp. 3091-3096, December 2003.

[10] E. R. Westervelt, J. W. Grizzle, and C. Canudas, "Switching and PI control of walking motions of planar biped walkers," IEEE Transactions on Automatic Control, vol. 48, issue 2, pp. 308-312, February 2003.

[11] J. W. Grizzle, "Remarks on event-based stabilization of periodic orbits in systems with impulse effects," Second International Symposium on Communications, Control and Signal Processing, 2006.

[12] E. R. Westervelt, G. Buche, and J. W. Grizzle, "Experimental validation of a framework for the design of controllers that induce stable walking in planar bipeds," The International Journal of Robotics Research, vol. 24, no. 6, pp. 559-582, June 2004.

[13] K. Sreenath, H. W. Park, J. W. Grizzle, "Design and experimental implementation of a compliant hybrid zero dynamics controller with active force control for running on MABEL," Proceedings of the 2012 IEEE International Conference on Robotics and Automation, Saint Paul, MN, pp. 51-56, May 2012.

[14] C. Chevallereau, J. W. Grizzle, and C. L. Shih, "Asymptotically stable walking of a five-link underactuated 3-D bipedal robot," IEEE Transactions on Robotics, vol. 25, issue 1, pp. 37-50, February 2009.

[15] K. Akbari Hamed, N. Sadati, W. A. Gruver, and G. A. Dumont, "Exponential stabilisation of periodic orbits for running of a threedimensional monopedal robot," IET Control Theory \& Applications, vol. 5, issue 11, pp. 1304-1320, 2011.

[16] A. Ramezani and J. W. Grizzle, "ATRIAS 2.0, a new 3D bipedal robotic walker and runner," Proceedings of the 2012 International Conference on Climbing and walking Robots and the Support Technologies for Mobile Machines, Baltimore, MD, pp. 467-474, July 2012.

[17] J. A. Grimes and J. W. Hurst, "The design of ATRIAS 1.0 a unique monoped, hopping robot," Proceedings of the 2012 International Conference on Climbing and walking Robots and the Support Technologies for Mobile Machines, Baltimore, MD, pp. 548-554, July 2012.

[18] D. D. Bainov and P. S. Simeonov, Systems with Impulse Effects: Stability, Theory and Applications, Ellis Horwood Limited, April 1989.

[19] H. Ye, A. N. Michel, and L. Hou, "Stability theory for hybrid dynamical systems," IEEE Transactions on Automatic Control, vol. 43, no. 4, pp. 461-474, 1998.

[20] M. C. de Oliveira, J. Bernussou, and J. C. Geromel, "A new discretetime robust stability condition," Systems \& Control Letters, vol. 37, pp. 261-265, 1999. 